\title{
Surname lists to identify South Asian and Chinese ethnicity from secondary data in Ontario, Canada: a validation study
}

\author{
Baiju R Shah*1,2,3, Maria Chiu1,2, Shubarna Amin², Meera Ramani2, Sharon Sadry² and Jack V Tu1,2,3
}

\begin{abstract}
Background: Surname lists are useful for identifying cohorts of ethnic minority patients from secondary data sources. This study sought to develop and validate lists to identify people of South Asian and Chinese origin.

Methods: Comprehensive lists of South Asian and Chinese surnames were reviewed to identify those that uniquely belonged to the ethnic minority group. Surnames that were common in other populations, communities or ethnic groups were specifically excluded. These surname lists were applied to the Registered Persons Database, a registry of the health card numbers assigned to all residents of the Canadian province of Ontario, so that all residents were assigned to South Asian ethnicity, Chinese ethnicity or the General Population. Ethnic assignment was validated against self-identified ethnicity through linkage with responses to the Canadian Community Health Survey.

Results: The final surname lists included 9,950 South Asian surnames and 1,133 Chinese surnames. All 16,688,384 current and former residents of Ontario were assigned to South Asian ethnicity, Chinese ethnicity or the General Population based on their surnames. Among 69,859 respondents to the Canadian Community Health Survey, both lists performed extremely well when compared against self-identified ethnicity: positive predictive value was $89.3 \%$ for the South Asian list, and $91.9 \%$ for the Chinese list. Because surnames shared with other ethnic groups were deliberately excluded from the lists, sensitivity was lower ( $50.4 \%$ and $80.2 \%$, respectively).

Conclusions: These surname lists can be used to identify cohorts of people with South Asian and Chinese origins from secondary data sources with a high degree of accuracy. These cohorts could then be used in epidemiologic and health service research studies of populations with South Asian and Chinese origins.
\end{abstract}

\section{Background}

Because many secondary data sources used in health research do not include information on race or ethnicity, surnames are often used as a proxy when studying health care for ethnic populations. In the United States, the Bureau of the Census compiled a list of "Spanish surnames" in the 1950s which has since been updated every decade [1]. These lists have been used extensively to study Hispanic populations using secondary data sources.

Canada's two largest minority populations are those with South Asian (from India, Pakistan, Bangladesh and Sri Lanka) and Chinese origins [2]. Surname lists to identify both of these population groups have been devel-

* Correspondence: baiju.shah@ices.on.ca

1 Institute for Clinical Evaluative Sciences, 2075 Bayview Avenue, Toronto, Ontario, M4N 3M5, Canada

Full list of author information is available at the end of the article oped. To identify South Asian populations, two proprietary computerized algorithms using first and surnames were developed in the United Kingdom $[3,4]$. The SANGRA algorithm yielded sensitivity from $89 \%$ to $96 \%$ versus self-reported ethnicity from various data sources across England, with positive predictive value between $80 \%$ and $89 \%$ [3]. In contrast, the Nam Pehchan program had a sensitivity of $61 \%$ and a positive predictive value of 97\% against self-reported ethnicity in a national sample [4]. In addition an American list of South Asian surnames was developed by Lauderdale and Kestenbaum [5]. However, it had only $38 \%$ sensitivity and $77 \%$ positive predictive value against census records. In addition, the US Census derived an Asian-Indian surname list from the 2000 Census [6], but the test characteristics of this list have not been evaluated. Sheth et al. [7] derived a list of 
South Asian surnames using Canadian death certificate data, by selecting people whose place of birth was South Asia or countries with large South Asian populations. They performed a telephone survey of 100 people who had surnames on the list and 100 who did not, and found that the list had a sensitivity of $96 \%$ versus self-reported ethnicity. However, this list did not specifically exclude surnames that are common to both South Asian and other populations, such as Khan, Ahmed, DeSouza or Fernandes. To identify Chinese populations, several surnames lists have been developed. In 1990, Hage et al. [8] developed a list of 145 Chinese surnames from the Melbourne, Australia telephone directory. In 1993, Choi et al. [9] published a list of over 200 Chinese surnames that was derived by finding the surnames of all people whose place of birth recorded in the Ontario vital statistics registry was China, Taiwan or Hong Kong. This list was further expanded and modified by Tjam [10] and by Quan et al. [11]. However, a limitation of most of these surname lists for both minority groups is that they are either unvalidated, or they are validated only in small settings rather than in a broad population, and so their generalizability to other contexts is uncertain.

The objective of this study was to develop and validate surname lists that could be used to identify cohorts of people with South Asian and Chinese origins from secondary data sources. The study was conducted in Ontario, Canada's largest province and also one of its most ethnically diverse. To identify such cohorts, we want to be confident that individuals identified using these surname lists truly come from the target ethnic population. Ensuring that such cohorts are representative of the entire ethnic population is secondary. Therefore, our goal was to maximize positive predictive value of the surname lists, potentially sacrificing sensitivity.

\section{Methods}

\section{Development of the surname lists}

To develop the South Asian surname list, we started with the previously developed Canadian list of South Asian surnames developed using death certificate data [7]. We added surnames found in community telephone directories and in an encyclopedia of surnames published by the Indian government [12]. Each name was then reviewed by at least two researchers with South Asian origins. Surnames were excluded if they were not felt to be uniquely South Asian (i.e., if the surname was common also in other populations, communities or ethnic groups). If there was disagreement between the researchers about whether or not to exclude a surname, it was reviewed by a panel of five researchers with South Asian origins until a consensus decision was reached. The final list included all surnames that were believed, by consensus, to be uniquely South Asian.
Although a comprehensive list of Chinese surnames has been previously published and validated, we noted that this list included some names that were not uniquely Chinese (e.g., Diep, Jain, Kang and Sen) [11]. As such, we were concerned about the positive predictive value of identifying cohorts of patients with Chinese origin using this list. Therefore, we repeated a similar consensus process for the surnames from that list, to create a final list of surnames believed, by consensus, to be uniquely Chinese.

\section{Administrative data sources}

The Institute for Clinical Evaluative Sciences (ICES) is a health services research organization funded in part by the Ontario Ministry of Health and Long-Term Care $(\mathrm{MOH})$ to conduct analyses of provincial health care administrative databases for policy-relevant and scientific research. One of these administrative data sources is the Registered Persons Database (RPDB), which is a registry of the assigned health card numbers for all current and former residents of the province of Ontario (current population $=13$ million). The nominal file received from the $\mathrm{MOH}$ includes patients' surnames. In the data sharing agreement between the $\mathrm{MOH}$ and ICES, only three named individuals have access to this nominal file. They anonymize it prior to its release for general analytical use by removing the names and by encrypting the health card numbers. Because this encryption uses a reproducible algorithm common to all of the administrative data sources, individuals can be linked between databases via this unique encrypted number.

The surnames lists were applied to the nominal RPDB file to create an ethnic identification file. All Ontario residents whose surnames were on the South Asian list were assigned to South Asian ethnicity; all residents whose surnames were on the Chinese list were assigned to Chinese ethnicity; all others were assigned to the General Population group. The final surname-derived ethnic identification file included both the encrypted health card number and the ethnicity assignment for all Ontario residents. (See Figure 1.)

\section{Validation}

We validated the surname lists against self-reported ethnicity from the Canadian Community Health Survey (CCHS). The CCHS is a recurring cross-sectional national telephone survey conducted by Statistics Canada, targeting household residents aged 12 or older, excluding those living on Indian Reserves, Canadian Forces Bases, institutions and some remote areas. Prior to 2007 , the survey operated on a two-year collection cycle, with the first year examining general population health in a large sample, and the second year focusing on specific health topics in a smaller sample. Each respondent in each survey is assigned a person-level weight, so that the 


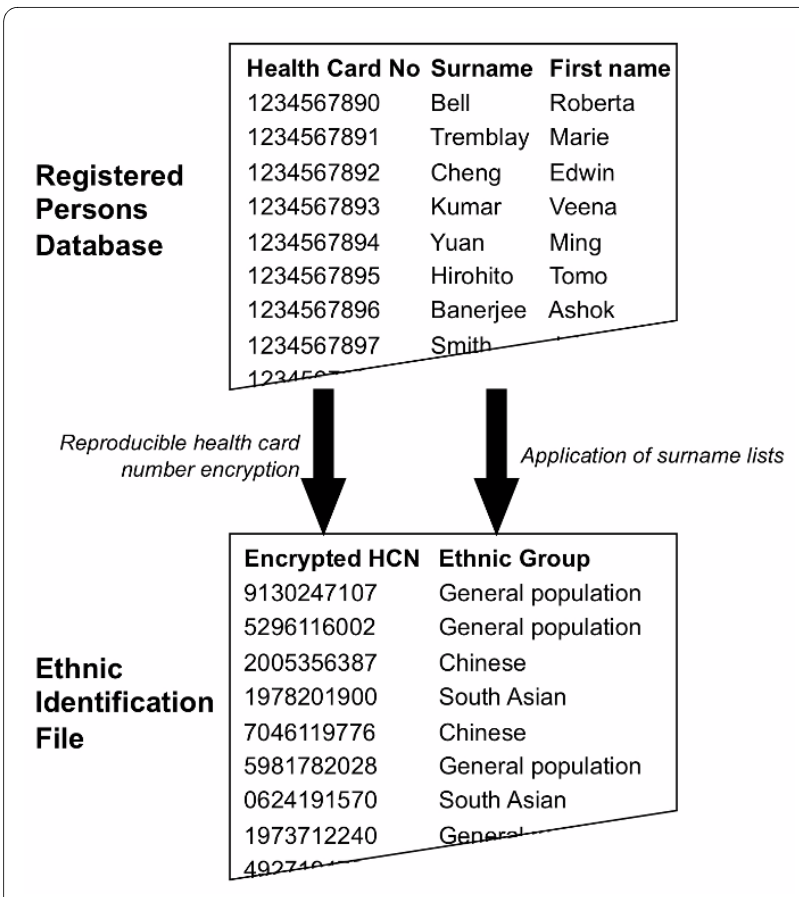

Figure 1 The derivation of the surname-derived ethnic identification file from the Registered Persons Database.

weight corresponds to the number of people in the entire population that are represented by that respondent, and the sum of all weights in one year's survey equals the population of Canada [13]. Three sampling frames are used for the survey, and the final weight assigned to an individual is integrated from the weights independently assigned from each sampling frame. Weights are also calibrated to account for other potential biases, including nonresponse and oversampling of households with multiple telephone lines. The use of the weights in analyses of these data is required to ensure findings are representative of the population, and not just of survey sample [13]. Although ethnicity is not specifically included in the derivation of respondents' weights, participants are selected at random from the population, so there is no reason to suspect that the weightings would systematically underor over-represent any ethnic group, particularly since the survey is administered in over 20 languages. The CCHS data set does not include respondents' surnames, but respondents did give permission to have their survey responses linked with health administrative data sources via their health card number. This was anonymized using the usual encryption algorithm at ICES, so that the CCHS data can be linked with the other administrative data sources, including the RPDB.

The gold standard was self-reported ethnicity from the CCHS. Two CCHS questions, which were routinely collected as part of the demographic profile of survey respondents, were used. These were "To what ethnic groups did your ancestors belong?" and "People living in Canada come from many different cultural and racial backgrounds. Are you...?" Multiple responses were permitted for each question. Those respondents who had a single response of "South Asian" to either question were assigned to South Asian ethnicity; those who had a single response of "Chinese" to either question were assigned to Chinese ethnicity; and all others were assigned to the General Population.

We studied all adult respondents to any of the 2001, 2002 or 2003 cycles of the CCHS. For each respondent, the gold-standard ethnicity was established from their self-reported responses to the survey, while their surname-derived ethnicity was derived through linkage of their encrypted health card number from the CCHS dataset with their ethnicity assignment in the surnamederived ethnic identification file. Validity of the South Asian surname list was determined by measuring sensitivity (the proportion of people self-identified as South Asian who were detected as such by the surname list), specificity (the proportion of people self-identified as not being South Asian who were detected as such by the surname list), positive predictive value (the proportion of those detected by the surname list as South Asian who self-identified as such) and negative predictive value (the proportion of those detected by the surname list as not being South Asian who self-identified as such). Similar calculations were made to validate the Chinese surname list. In these calculations, each respondent was weighted by his or her person-level weight (divided by 3 , because we were combining respondents from three survey years). We compared the previously-published Chinese surname list [11] with our Chinese surname list by comparing surname-derived ethnicity using both lists against the same gold standard. Finally, we compared the positive predictive value of each of our surname lists stratified by sex, age and immigration status.

\section{Ethics}

The data for the study are not publicly available, but were provided to ICES by the $\mathrm{MOH}$ under a research agreement. ICES is permitted to hold, link and analyze these data for research purposes as a named "prescribed entity" in Ontario's health information privacy law, the Health Information Protection Act [14]. The study was approved by the institutional review board of Sunnybrook Health Sciences Centre.

\section{Results}

The initial list of potentially South Asian surnames included 13,949 names to be screened. Of them, 9,950 were selected by consensus for the final list of uniquely South Asian surnames. From the initial 1,185 Chinese surnames, 1,133 were selected for the final list. Each sur- 
name list was linked with the nominal RPDB, and all $16,688,384$ current and former residents of Ontario were assigned to South Asian ethnicity $(500,807)$, Chinese ethnicity $(808,670)$ or the General Population $(15,378,907)$. The 200 most common surnames from each group are presented in Table 1.

There were 69,859 CCHS respondents who were included in this validation study, of whom 1,400 selfidentified as South Asian (5.4\% of the weighted population), and 1,129 self-identified as Chinese (4.0\% of the weighted population). The baseline characteristics of the population are shown in Table 2 . The sensitivity, specificity and positive and negative predictive values of the South Asian and Chinese surnames lists are shown in Table 3. Both lists perform extremely well, with approximately $90 \%$ positive predictive value. The sensitivity of the South Asian list was low, as expected, since the many surnames common for but not unique to South Asians were excluded from the final list. Compared to the previously published Chinese list, our modified Chinese surname list had slightly better positive predictive value with the sacrifice of a small amount of sensitivity.

Table 4 shows the positive predictive value of the surname lists among patients stratified by sex, age and immigration status. The surname list was slightly less likely to accurately predict self-identified ethnicity among South Asian women, but the difference between sexes was not marked among people with Chinese origin. As expected, longer time since immigration was associated with worse positive predictive value in both ethnic groups. Both lists performed poorly among those individuals who were Canadian-born.

\section{Discussion}

We have developed surname lists that can accurately identify people with self-reported South Asian or Chinese origins through secondary data sources. Despite the relatively low prevalence of minorities in the total population, both lists had excellent positive predictive value [15], so they can be used to confidently assemble ethnic minority cohorts for epidemiologic or health services research studies of people with South Asian or Chinese origins. However, the South Asian list had, as expected, low sensitivity. Therefore, it cannot be used, for example, to determine the proportion of a disease cohort that has South Asian origins. The Chinese list had a slightly better positive predictive value than the previously published list on which is was based [11], and also retained good sensitivity.

The surname lists showed markedly poorer positive predictive value for people who were Canadian-born. This most likely reflects people from other backgrounds who have adopted an ethnic surname through marriage, or to people whose parents were from different ethnic backgrounds. (However, it is noteworthy that people of South Asians and Chinese origins are the least likely in Canada to report being married to someone from outside their ethnic group [2].) It may also indicate that Canadian-born minorities may not self-identify with their ethnic group. Hence, although the surname lists are useful in the overall population, they may be less valid for use in this specific subgroup. In the future, as more people adopt surnames from other ethnic groups and as more people with multiple ethnic origins are born, the usefulness of surname lists to identify ethnic populations from secondary data may decline.

Identification of Chinese surnames is relatively straightforward, as about $95 \%$ of the Chinese population is Han Chinese. There are only a few hundred different common surnames in Chinese script [16], and a limited number of Westernized spellings of each name. In contrast, the South Asian population includes a large number of language, religious and cultural groups, each with a multitude of common surnames. Hence, our original list of South Asian surnames to screen was more than 10 times longer than the list of Chinese surnames. Because we sought to maximize positive predictive value by limiting our final list to include only those names that were uniquely South Asian, the final list includes mostly Hindu, Sikh and Sri Lankan surnames. Names used by South Asian Muslims or Christians are shared with people from those faiths in other parts of the world, and indeed may be more common in people with other ethnic backgrounds (e.g., Arab, Persian or Portuguese). Although including these names could have improved the sensitivity of our surname list, their inclusion would have led to an unacceptably dramatic drop in positive predictive value, and would have rendered the surname list invalid for our purposes. Other South Asian name algorithms have included such names to maximize sensitivity, but this came at a cost of very poor positive predictive value [17].

There are limitations to this methodology. As noted above, the included surnames on the South Asian list are mostly Hindu, Sikh and Sri Lankan names while surnames from other religious or cultural groups are excluded. Since there is evidence of biological and behavioural heterogeneity between South Asian populations [18-21], any cohorts built using the surname list may therefore systematically exclude people with different biological or clinical risks. The same is true for the Chinese list, although the Han population does make up an overwhelming majority of the Chinese population. In addition, there are limitations to the gold standard. We restricted our gold-standard definition to questions about ancestry and cultural/racial background. We did not use survey questions about place of birth or first language as these may not be specific nor sensitive for ethnicity. 
Table 1: The 200 most common surnames from the South Asian and Chinese surname lists, and from the general population in Ontario.

\begin{tabular}{|c|c|c|c|c|c|}
\hline \multicolumn{2}{|l|}{ South Asian } & \multicolumn{2}{|c|}{ Chinese } & \multicolumn{2}{|c|}{ General population } \\
\hline Name & $\mathbf{N}$ & Name & $\mathbf{N}$ & Name & $\mathbf{N}$ \\
\hline Patel & 35,984 & Wong & 34,567 & Smith & 91,575 \\
\hline Singh & 31,820 & Chan & 32,692 & Brown & 57,222 \\
\hline Sharma & 10,216 & $\mathrm{Li}$ & 27,608 & Lee & 49,898 \\
\hline Kaur & 7,462 & Chen & 25,618 & Wilson & 43,803 \\
\hline Persaud & 6,982 & Wang & 22,548 & Martin & 38,878 \\
\hline Grewal & 6,044 & Zhang & 18,003 & Campbell & 34,551 \\
\hline Sidhu & 5,862 & Lam & 15,910 & Williams & 34,104 \\
\hline Dhaliwal & 5,209 & Leung & 13,696 & Thompson & 33,810 \\
\hline Dhillon & 4,847 & $\mathrm{Ho}$ & 12,830 & Jones & 32,644 \\
\hline
\end{tabular}

Agarwal, Aggarwal, Ahluwalia, Ahuja, Akhtar, Akhter, Akram, Anand, Arora, Arumugam, Atwal, Aujla, Aulakh, Bains, Bajwa, Baksh, Balachandran, Balasingam, Balasubramaniam, Banerjee, Bansal, Banwait, Bedi, Begum, Beharry, Bhalla, Bhandari, Bhardwaj, Bhatia, Bhatt, Bhatti, Bhavsar, Bhogal, Bhullar, Boodram, Boparai, Brar, Chadha, Chahal, Chand, Chandra, Chaudhary, Chaudhry, Chauhan Chawla, Cheema, Chohan, Chopra, Choudhry, Choudhury, Chowdhury, Das, Dass, Datta, Deol, Desai, Dhami, Dhanoa, Dhindsa, Dosanjh, Gandhi, Ganesh, Garcha, Ghosh, Ghuman, Gopaul, Gupta, Heer, Hundal, Jaffer, Jafri, Jain, Jassal, Johal, Joshi, Kahlon, Kalra, Kanagaratnam, Kandasamy, Kandiah, Kanji, Kapadia, Kapoor, Karam, Karimi, Kaushal, Khaira, Khanna, Khatri, Khokhar, Kohli, Kumar, Kumarasamy, Ladha, Lakhani, Lal, Lalani, Lall, Mahabir, Mahadeo, Maharaj, Mahendran, Malhi, Malhotra, Mangat, Manji, Manoharan, Maraj, Matharu, Mathur, Mehta, Mistry, Modi, Mohan, Multani, Nadarajah, Naik, Nair, Naraine, Navaratnam, Nijjar, Panchal, Pandher, Pandya, Panesar, Pannu, Parekh, Parikh, Parmar, Parveen, Pathak, Pathan, Pathmanathan, Persad, Prajapati, Prasad, Prashad, Purewal, Puri, Rai, Raja, Rajaratnam, Rajkumar, Ram, Ramcharan, Ramkissoon, Ramnarine, Rampersad,

Rampersaud, Ramroop, Randhawa, Rao, Sahota, Saini, Samra, Sangha, Sanghera, Sankar, Sehgal, Sekhon, Selvarajah, Selvaratnam, Sethi, Shanmuganathan, Shergill, Sheth, Shukla, Sinha, Sinnathamby, Sivakumar, Sivasubramaniam, Sodhi, Sohail, Sohal, Sohi, Sood, Sritharan, Subramaniam, Tharmalingam, Thind, Toor, Trahan, Trivedi, Uppal, Varghese, Verma, Virdi, Virk, Vyas, Walia
An, Au, Bai, Cai, Cao, Chang, Chao, Chau, Cheng, Cheong, Cheung, Chiang, Chin, Ching, Chiu, Cho, Chong, Chou, Chow, Choy, Chu, Chua, Chui, Chun, Chung, Cui, Dai, Deng, Ding, Dong, Du, Duong, Eng, Fan, Fang, Feng, Fok, Fong, Fu, Fung, Gao, Gong, Gu, Guan, Guo, Ha, Han, He, Hong, $\mathrm{Hou}, \mathrm{Hsu}, \mathrm{Hu}$, Hua, Huang, Hui, Hum, Hung, Hwang, Ing, Ip, Jang, Ji, Jia, Jiang, Jin, Kam, Kan, Ko, Kong, Koo, Ku, Kung, Kuo, Kwan, Kwok, Kwon, Kwong, La, Lai, Lao, Lau, Lei, Leong, Liang, Liao, Lin, Ling, Lo, Lu, Lui, Luk, Lum, Luo, Luong, Ma, Mah, Mai, Mak, Man, Mao, Mei, Meng, Mian, Mo Mok, Monk, Ng, Ngai, Ong, Ou, Pan, Pang, Peng, Phung, Poon, Qi, Qian, Qin, Qiu, Quan, Ren, Seto, Shao, Shen, Shi, Shum, Sin, Situ, Siu, So, Song, Su, Sun, Sung, Szeto, Ta, Tai, Tam, Tan, Tang, Tao, Tian, To, Tom, Tong, Tsai, Tsang, Tse, Tsui, Tu Tung, Wan, Wei, Wen, Wing, Woo, Wu, Xia, Xiao, Xie, Xu, Xue, Yan, Yang, Yao, Yap, Yau, Ye, Yee, Yeh, Yeung, Yi, Yim, Yin, Yip, Yiu, Yong, Yoon, Yu, Yuan, Yue, Yuen, Yung, Zeng, Zhao, Zheng, Zhong, Zhou, Zhu, Zou
Adams, Ahmed, Alexander, Ali, Allen, Anderson, Andrews, Armstrong, Bailey, Baker, Barnes, Bélanger, Bell, Bennett, Black, Boyd, Bradley, Brooks, Burke, Burns, Butler, Cameron, Carter, Chapman, Choi, Clark, Clarke, Cole, Collins, Cook, Cooper, Cox, Craig, Crawford, Cunningham, Da Silva, Davidson, Davies, Davis, Dawson, Dixon, Douglas, Doyle, Duncan, Dunn, Edwards, Elliott, Ellis, Evans, Ferguson, Fernandes, Ferreira, Fisher, Fleming, Ford, Foster, Fox, Francis, Fraser, Gagnon, Garcia, Gauthier, George, Gibson, Gill, Gordon, Graham, Grant, Gray, Green, Hall, Hamilton, Harris, Harrison, Hart, Harvey, Hassan, Hayes, Henderson, Henry, Hill, Holmes, Howard, Hughes, Hunt, Hunter, Huynh, Jackson, James, Johnson, Johnston, Kelly, Kennedy, Kerr, Khan, Kim, King, Knight, Lalonde, Lawrence, Le, Leblanc, Lewis, Little, Macdonald, Mackenzie, Maclean, Macleod, Mann, Marshall, Mason, Matthews, McDonald, McIntyre, McKay, McKenzie, McLean, McLeod, Miller, Mills, Mitchell, Mohamed, Moore, Morgan, Morin, Morris, Morrison, Murphy, Murray, Nelson, Nguyen, O'Brien, Palmer, Park, Parker, Parsons, Patterson, Paul, Payne, Pereira, Perry, Peters, Phillips, Porter, Powell, Price, Reid, Reynolds, Richards, Richardson, Roberts, Robertson, Robinson, Rogers, Rose, Ross, Roy, Russell, Ryan, Santos, Saunders, Scott, Seguin, Shah, Shaw, Silva, Simpson, Spencer, Stevens, Stevenson, Stewart, Sullivan, Sutherland, Thomas, Thomson, Tran, Tremblay, Turner, Walker, Wallace, Walsh, Ward, Warren, Watson, White, Williamson, Wood, Woods, Wright, Young 
Table 2: Unweighted and weighted baseline characteristics of the study population

\begin{tabular}{|c|c|c|c|}
\hline Characteristic & South Asian & Chinese & General population \\
\hline \multicolumn{4}{|l|}{ Unweighted } \\
\hline $\mathrm{N}$ & 1,400 & 1,129 & 67,330 \\
\hline \multicolumn{4}{|l|}{ Surname-derived ethnicity } \\
\hline South Asian & 654 & 4 & 129 \\
\hline Chinese & 9 & 899 & 139 \\
\hline General population & 737 & 226 & 67,062 \\
\hline \multicolumn{4}{|l|}{ Weighted } \\
\hline Proportion of the population & $5.4 \%$ & $4.0 \%$ & $90.6 \%$ \\
\hline \multicolumn{4}{|l|}{ Sex } \\
\hline Male & $54.7 \%$ & $52.5 \%$ & $48.4 \%$ \\
\hline Female & $45.3 \%$ & $47.5 \%$ & $51.6 \%$ \\
\hline \multicolumn{4}{|l|}{ Age } \\
\hline 44 or younger & $64.4 \%$ & $62.6 \%$ & $52.0 \%$ \\
\hline 45 to 64 & $29.2 \%$ & $27.6 \%$ & $31.4 \%$ \\
\hline 65 or older & $6.4 \%$ & $9.8 \%$ & $16.6 \%$ \\
\hline \multicolumn{4}{|l|}{ Immigration status } \\
\hline Born in Canada & $7.0 \%$ & $9.6 \%$ & $73.7 \%$ \\
\hline Immigrant $\leq 10$ years & $47.2 \%$ & $38.1 \%$ & $6.0 \%$ \\
\hline Immigrant 11 to 20 years & $25.9 \%$ & $28.3 \%$ & $4.8 \%$ \\
\hline Immigrant $>20$ years & $19.9 \%$ & $24.0 \%$ & $15.5 \%$ \\
\hline
\end{tabular}

There may have been confusion about the meaning or intent of the survey questions, which might have led to incorrect responses. In particular, terms like "South Asian" (versus "Southeast Asian") or "Indian" (versus "Aboriginal") may have been confused by both respondents and interviewers. Finally, generalizing surname lists to populations outside of the areas in which they were derived is problematic, because of differing immigration patterns to different parts of the world. This phenomenon was demonstrated with the Nam Pehchan algorithm in the United Kingdom, which had a sensitivity of over 94\% when evaluated for individuals in Bradford where it was developed, but only $61 \%$ when evaluated for individuals from across England [4]. Therefore, our surname list

Table 3: Test characteristics of the South Asian and Chinese surname lists compared against self-reported ethnicity

\begin{tabular}{lccc}
\hline Surname list & Sensitivity (\%) & $\begin{array}{c}\text { Specificity (\%) } \\
\text { vesitive predictive } \\
\text { value (\%) }\end{array}$ & $\begin{array}{c}\text { Pegative predictive } \\
\text { value }\end{array}$ \\
\hline South Asian & 50.4 & 99.7 & 89.3 \\
\hline $\begin{array}{l}\text { Chinese } \\
\text { vreviously published } \\
\text { Chinese }\end{array}$ & 80.2 & 99.7 & 91.9 \\
\hline
\end{tabular}


Table 4: Positive predictive value (\%) of the South Asian and Chinese surname lists compared against self-reported ethnicity, stratified by sex, age and immigration status.

\begin{tabular}{|c|c|c|}
\hline Characteristic & South Asian & Chinese \\
\hline Overall & 89.3 & 91.9 \\
\hline \multicolumn{3}{|l|}{ Sex } \\
\hline Male & 91.4 & 92.1 \\
\hline Female & 86.5 & 91.7 \\
\hline \multicolumn{3}{|l|}{ Age } \\
\hline 44 or younger & 89.2 & 92.6 \\
\hline 45 to 64 & 88.0 & 90.0 \\
\hline 65 or older & 94.6 & 93.0 \\
\hline \multicolumn{3}{|l|}{ Immigration status } \\
\hline Born in Canada & 58.7 & 77.8 \\
\hline Immigrant $\leq 10$ years & 95.2 & 95.9 \\
\hline Immigrant 11 to 20 years & 89.6 & 94.1 \\
\hline Immigrant $>20$ years & 88.6 & 88.1 \\
\hline
\end{tabular}

would likely not have the same performance in other jurisdictions.

\section{Conclusions}

In conclusion, these surname lists can be used to accurately identify cohorts of people with South Asian and Chinese origins from secondary data sources, although these cohorts would, by necessity, not always be comprehensive or representative of the entire South Asian or Chinese population.

\section{Abbreviations}

CCHS: Canadian Community Health Survey; ICES: Institute for Clinical Evaluative Sciences; RPDB: Registered Persons Database.

\section{Competing interests}

The authors declare that they have no competing interests.

\section{Authors' contributions}

BRS conceived of the study, participated in its design and in the surname review, performed the statistical analyses, and drafted the manuscript. MC participated in the design of the study and the surname review. SA, MR and SS all participated in the surname review. JVT participated in the design of the study. All authors revised the manuscript critically for intellectual content, and all have read and approved the final version.

\section{Acknowledgements}

The authors wish to thank Nelson Chong, Nadia Gunraj and Sonia Anand for their assistance with the study. This project was funded by the Heart and Stroke Foundation of Ontario. Dr. Shah is supported by the Canadian Institutes of Health Research, the Canadian Diabetes Association and the Banting and Best Diabetes Centre of the University of Toronto. Ms Chiu is supported by a Canadian Institutes of Health Research Frederick Banting and Charles Best Canada Graduate Scholarship. Dr. Tu is supported by a Canada Research Chair in health services research, and a career investigator award from the Heart and
Stroke Foundation of Ontario. The Institute for Clinical Evaluative Sciences is supported in part by the Ontario Ministry of Health and Long-Term Care.

\section{Author Details}

'Institute for Clinical Evaluative Sciences, 2075 Bayview Avenue, Toronto, Ontario, M4N 3M5, Canada, 2 University of Toronto, Toronto, Ontario, Canada and ${ }^{3}$ Department of Medicine, Sunnybrook Health Sciences Centre, 2075 Bayview Avenue, Toronto, Ontario, M4N 3M5, Canada

Received: 9 March 2010 Accepted: 15 May 2010

Published: 15 May 2010

References

1. Word DL, Perkins RC: Building a Spanish surname list for the 1990's: a new approach to an old problem Washington, DC, U.S. Census Bureau; 1996.

2. Canada Statistics: Canada's Ethnocultural Mosaic, 2006 Census. Ottawa 2008

3. Nanchahal K, Mangtani P, Alston M, dos Santos Silva I: Development and validation of a computerized South Asian Names and Group Recognition Algorithm (SANGRA) for use in British health-related studies. J Public Health Med 2001, 23:278-285.

4. Harding S, Dews H, Simpson SL: The potential to identify South Asians using a computerised algorithm to classify names. Popul Trends 1999, 97:46-49.

5. Lauderdale DS, Kestenbau B: Asian American ethnic identification by surname. Popul Res Policy Rev 2000, 19:283-300.

6. Falkenstein MR: The Asian and Pacific Islander surname list: as developed from Census 2000 [abstract]. Joint Statistical Meetings 2002 New York 2002.

7. Sheth T, Nargundkar M, Chagani K, Anand S, Nair C, Yusuf S: Classifying ethnicity utilizing the Canadian Mortality Data Base. Ethnic Health 1997, 2:287-295

8. Hage BH-H, Oliver RG, Powles JW, Wahlqvist ML: Telephone directory listings of presumptive Chinese surnames: an appropriate sampling frame for a dispersed population with characteristic surnames. Epidemiology 1990, 1:405-407.

9. Choi BCK, Hanley AJG, Holowaty EJ, Dale D: Use of surnames to identify individuals of Chinese ancestry. Am J Epidemiol 1993, 138:723-734 
10. Tjam EY: How to find Chinese research participants: use of a phonologically based surname search method. Can J Public Health 2001, 92:138-142.

11. Quan H, Wang F, Schopflocher D, Norris C, Galbraith PD, Faris P, Graham MM, Knudtson ML, Ghali WA: Development and validation of a surname list to define Chinese ethnicity. Med Care 2006, 44:328-333.

12. Singh KS: People of India: National Series Volume VIII: Communities, Segments, Synonyms, Surnames and Titles Delhi, Oxford University Press, 1996.

13. Canadian Community Health Survey (CCHS). 2007 microdata files user guide [http://www.statcan.gc.ca/imdb-bmdi/document/ 3226 D7 T9 V4-eng.pdf]

14. Ontario.Legislative Assembly: Health Information Protection Act, 2004. 2004. Bill 31, 38th Legislature, 1st Session

15. Loong TW: Understanding sensitivity and specificity with the right side of the brain. BMJ 2003, 327:716-719.

16. Hundred Family Surnames [http://en.wikipedia.org/wiki/ Hundred Family Surnames]

17. Cummins C, Winter H, Cheng KK, Maric R, Silcocks P, Varghese C: An assessment of the Nam Pehchan computer program for the identification of names of South Asian ethnic origin. J Public Health 1999, 21:401-406

18. Balarajan R, Bulusu L, Adelstein AM, Shukla V: Patterns of mortality among migrants to England and Wales from the Indian subcontinent. BMJ 1984, 289:1185-1187.

19. Ramaiya KL, Swai ABM, McLarty DG, Bhopal RS, Alberti KGMM: Prevalences of diabetes and cardiovascular disease risk factors in Hindu Indian subcommunities in Tanzania. BMJ 1991, 303:271-276.

20. McKeigue PM, Shah B, Marmot MG: Relation of central obesity and insulin resistance with high diabetes prevalence and cardiovascular risk in South Asians. Lancet 1991, 337:382-386.

21. Bhopal R, Unwin N, White M, Yallop J, Walker L, Alberti KGMM, Harland J, Patel S, Ahmad N, Turner C, et al: Heterogeneity of coronary heart disease risk factors in Indian, Pakistani, Bangladeshi, and European origin populations: cross sectional study. BMJ 1999, 319:215-220.

\section{Pre-publication history}

The pre-publication history for this paper can be accessed here: http://www.biomedcentral.com/1471-2288/10/42/prepub

doi: $10.1186 / 1471-2288-10-42$

Cite this article as: Shah et al., Surname lists to identify South Asian and Chinese ethnicity from secondary data in Ontario, Canada: a validation study BMC Medical Research Methodology 2010, 10:42

Submit your next manuscript to BioMed Centra and take full advantage of:

- Convenient online submission

- Thorough peer review

- No space constraints or color figure charges

- Immediate publication on acceptance

- Inclusion in PubMed, CAS, Scopus and Google Scholar

- Research which is freely available for redistribution

Submit your manuscript at www.biomedcentral.com/submit
C Biomed Central 